\title{
Megacolon Concurrent with Perineal Hernia in a Male Rough Collie
}

\author{
1,2* Okene, I. A., ${ }^{1}$ Che Mat Ariffin, N., ${ }^{1}$ Shaari, R and ${ }^{3}$ Budi Pramono, D, A.
}

${ }^{1}$ Faculty of Veterinary Medicine, University Malaysia Kelantan, City Campus, 16150 Kota Bharu, Kelantan, Malaysia

${ }^{2}$ Department of Veterinary Surgery and Radiology, Faculty of Veterinary Medicine, University of Maiduguri, 600244 Maiduguri, Nigeria

${ }^{3}$ Rumah Sakit Hewan Prof. Soeparwi, Universitas Gadjah Mada Jl. Yacaranda, Sekip Unit II, Yogyakarta 55281, Indonesia

*Author for Correspondence: ibrahim.az@umk.edu.my

\begin{abstract}
Perineal hernia occurs frequently in aged collies as a result of weakening of the pelvic diaphragmatic muscle or its total failure, thereby allowing displacement of abdominal contents into the perineum. Surgical management include subtotal colectomy, colotomy, internal obturator muscle transposition and perineal herniorrhaphy. This report highlights a case of perineal hernia associated with megacolon as well as its successful management in a dog. A seven years old, male Rough Collie was presented with a complaint of right perineal swelling that has lasted for a week as well as inappetance and absence of defecation. Physical examination was unremarkable except for 3\% dehydration and a firm, large perineal non-reducible swelling. Plain abdominal radiography revealed perineal hernia with megacolon. Serum chemistry showed azotaemia. Emergency surgical intervention involving colotomy, perineal herniorrhaphy with incisional colopexy were conducted. Post-operative care included ceftriaxone injection at $50 \mathrm{mg} / \mathrm{kg}$ intramuscularly, intravenous fluid infusion, one week fasting, daily wound dressing, Nutriplus ${ }^{\circledR}$ gel supplementation and administration of recovery diet post-fasting. Thus, surgical management using traditional perineal herniorrhaphy, colotomy with colopexy was effective in correcting the perineal hernia with megacolon.
\end{abstract}

Keywords: Colopexy; Colostomy; Megacolon; Perineal hernia; Perineal herniorrhaphy

\section{INTRODUCTION}

Megacolon is a persistently increased large intestinal diameter and hypomotility associated with severe constipation, or obstipation (Prokić et al., 2010). It is prevalent in non-castrated male dogs aged $7-9$ years old and may occur primarily or secondary to megacolon; or viceversa thereby leading to constipation and swelling lateral to the anus. Perineal hernia results from failure of the pelvic muscle diaphragmatic structures to support the pelvic and caudal abdominal contents, which are the rectum, bladder, and small intestine (Vnuk et al., 2008; Lee, 2012). The distended colon pushes against the pelvic diaphragm muscles, resulting in the separation of the muscles, formation of hernia ring and herniation of abdominal viscera into the perineum. Herniated colon results into perineal swelling, intestinal blockage, constipation, obstipation, and further distension of preceding segments of the colon. The distended colon often imparts pressure on abdominal contents, such as the urinary bladder thereby causing obstructive post-renal uraemia (Prokić et al., 2010). Surgical management is imperative with included subtotal colectomy, colotomy, internal obturator muscle transposition and perineal herniorrhaphy. This report highlights the successful surgical management of perineal hernia associated with megacolon in a dog.

\section{CASE MANAGEMENT}

\section{Case History}

A seven-year-old intact male Rough Collie with up-to-date deworming and vaccination history was presented to Rumah Sakit Haiwan, Indonesia. The complaint was of right perineal swelling and history of 1-week long inappetence as well as the absence of defecation.

\section{Clinical Manifestation and Investigation}

Physical examination revealed intestinal hypomotility, distension, and presence of a segmented hard faecal mass within the intestine. Plain radiograph of the left lateral view of the abdomen (Figure 1) revealed perineal hernia containing a segment of the colon filled with faeces. A remarkably distended (unherniated) large colon was also observed to contain faecolith indicative of megacolon.

Haematology showed monocytos is indicative of the progression of the condition into the subacute stage as well as marginal erythrocytosis. Serum biochemistry showed normal hepatic enzyme values and presence of azotaemia (Table 1) Pressure imparted by the distended colon onto the urinary bladder may be responsible for the urinary tract obstruction and early onset of urine stasis and consequently backflow to the kidneys. Urine stasis and impaired outflow of urine from kidney result into reduced glomerular filtration rate (GFR). 
Table 1: Serum biochemistry values in a male Rough Collie indicating azotaemia

\begin{tabular}{llll}
\hline Parameter & Result & Reference values & Remark \\
\hline Alanine transferase & 40 & $0-51$ & \\
Alkaline phosphatase & 26 & $0-39$ & \\
Blood Urea Nitrogen & 19.6 & $5-18$ & Azotaemia \\
Creatinine & 1.2 & $<0.53$ & \\
\hline
\end{tabular}

Presence of colonic entrapment with distension of colonic wall by impacted faecal mass and urinary tract obstruction always warrants an emergency surgical intervention (Rayhanabad et al., 2009).

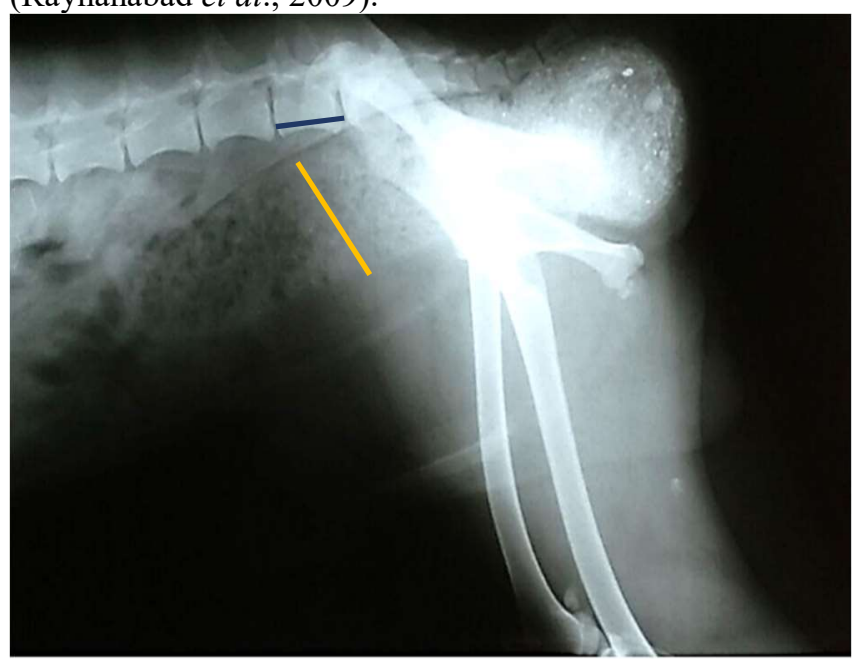

Figure 1: Left lateral view abdominal radiograph showing megacolon (luminal diameter - yellow line - is three times the length of L7 - blue line) with perineal hernia containing colon with faecal content.

\section{Management}

Subcutaneous injection of atropine sulphate $(0.1 \mathrm{mg} / \mathrm{kg})$ was administered as premedication. The right perineum area was prepped. General anaesthesia was induced with propofol (4 $\mathrm{mg} / \mathrm{kg}, \mathrm{IV}$ ) and maintained on isoflurane $1-2 \%$. With the patient on left lateral recumbency, a skin incision was made over the hernia sac. The skin and subcutaneous tissue were bluntly undermined with Metzenbaum scissors, followed by retraction and evaluation of the entrapped sigmoid colon. Colotomy was done via a longitudinal incision over the faecal mass on the antimesenteric border of the sigmoid colon which allowed for the removal of impacted faecal material which weighed $2 \mathrm{~kg}$. Everted mucosa was trimmed to make the edge even with that of the serosa. Colon was sutured with a single layer of simple interrupted suture of 3-0 polyglycolide absorbable material. The sutured colon was pushed back into the abdominal cavity through the perineal hernia ring (Figure 2).

The perineal hernia was corrected with simple continuous absorbable suture pattern. The suture began from the internal obturator muscle ventrolateral to the external anal sphincter and directed medially. The sacrotuberous ligament, the lateral aspects of the coccygeal and levator ani muscles were sutured to the medial aspect of the external anal sphincter using 3-0 monofilament non-absorbable nylon in a simple interrupted pattern. The subcutaneous tissue and skin were closed routinely (Figure 3). Colotomy was performed through a ventral midline abdominal approach to remove the remaining impacted faeces from the large intestine. It involved an incisional colopexy through a $4 \mathrm{~cm}$ longitudinal sero muscular incision made along the antimesenteric border of the colon. A second $3 \mathrm{~cm}$ long incision was made into the peritoneum and underlying musculature of the left abdominal wall. The edges of the seromuscular incision of the colon were then apposed to the edges of the abdominal wall incision with 3-0 non-absorbable nylon sutures. Subcutaneous tissue and skin were closed routinely.

Post-operative care included administration of Ceftriaxone sodium at $50 \mathrm{mg} / \mathrm{kg}(2 \mathrm{~mL})$ twice a day intramuscularly. The animal was fasted for one-week post-surgery, while the fluid balance was maintained with an intravenous infusion of normal saline. Nutriplus gel ${ }^{\circledR}$ (Virbac, Australia) supplementation was given orally, and resumption of feeding with i/d Royal Canin ${ }^{\circledR}$ (Indonesia) diet followed in the second week post-surgery.

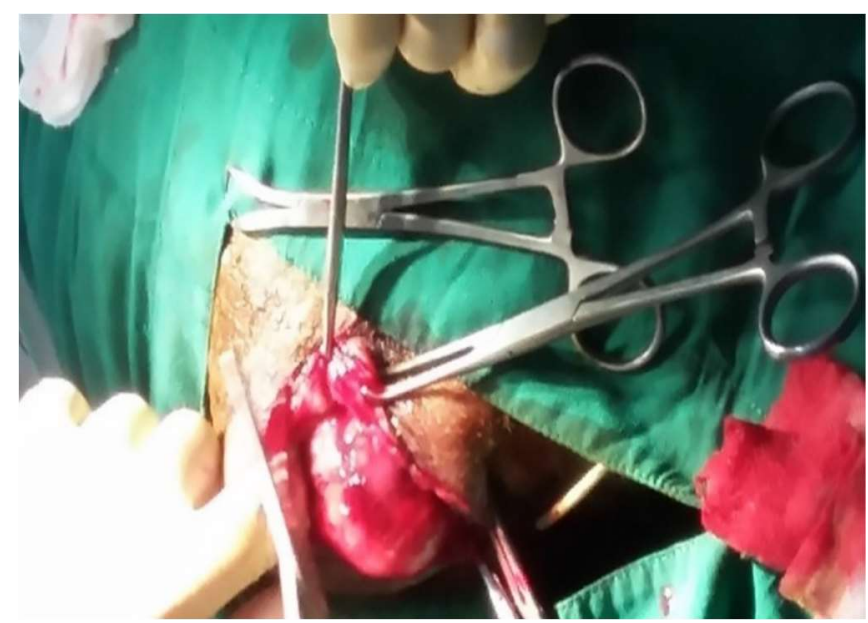

Figure 2: Skin incision and undermining of the surrounding subcutaneous tissue to expose herniated sigmoid colon.

\section{DISCUSSION}

Perineal herniorrhaphy, colostomy and colopexy were efficient in the successful resolution of the condition with the surgical site healing within seven days after surgery. Normal intestinal motility (on auscultation) and defecation resumed a day after the resumption of feeding.

In this case, we have observed the ability of megacolon to exacerbate herniation of the perineum by exertional pressure on the perineal muscles due to colonic enlargement. Most perineal hernias occur between the levator ani, external anal sphincter, and internal obturator muscles. However, in some cases, herniation occurs between the sacrotuberous ligament and coccygeus muscle, levator ani and coccygeus muscles or ischiourethralis, bulbocavernosus, and ischiocavernosus muscles (Machado et al., 2020). 


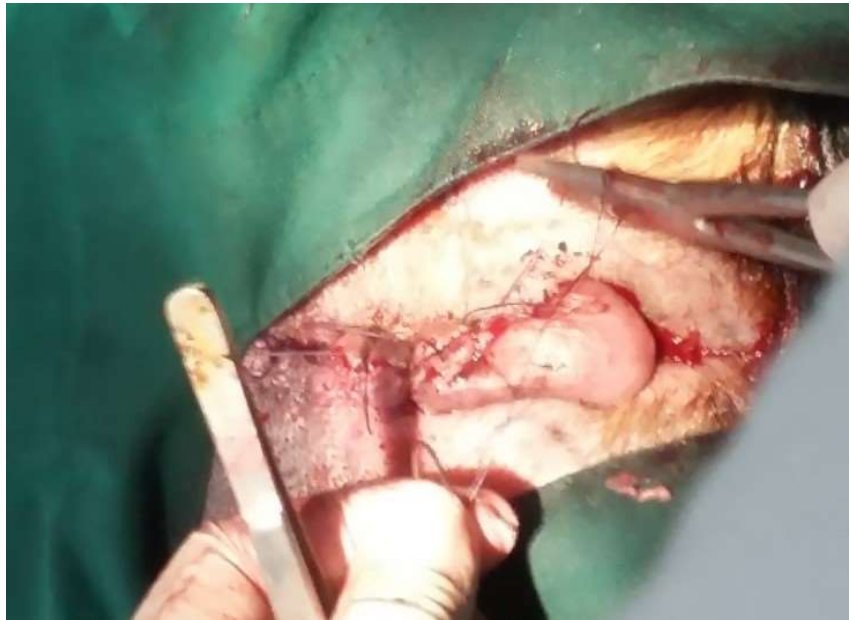

Figure 3: Routine skin closure using simple interrupted nonabsorbable suture pattern.

Herniation develops after the deterioration of the supporting pelvic diaphragm due to pressure from the impacted colon, causing separation of the external anal sphincter from the pelvic diaphragm muscles. This causes the loss of rectal wall support due to the increased pressure in the abdominal cavity. The abdominal viscera are displaced through the opening between the external anal sphincter and the other pelvic diaphragm muscles. In this case, some part of the colon has pushed through the opening and became trapped next to the rectum (Rayhanabad et al., 2009). As this organ gets trapped, faeces formed became entrapped in the region of the hernia sac and is unable to be evacuated physiologically. Overtime, the colon became dilated, and megacolon developed. The distended colon imparts pressure against the urinary bladder leading to urinary tract obstruction and urine stasis. Impaired urine outflow from the kidney (and possibly backflow from the obstructed urinary bladder) causes reduced glomerular filtration leading to post-renal uraemia as evidenced by high serum creatinine and blood urea nitrogen values (Segev et al., 2013). These values reverted to normal following the surgical correction described earlier.

Several surgical approaches are available for the treatment of perineal hernia such as the transabdominal and perineal approach (Fossum, 2018). In this case, the hernia was repaired post-colotomy using the right perineum because the diameter of the herniated sigmoid colon exceeded the diameter of the hernia ring, thereby ruling out a transabdominal approach. The transabdominal approach may be performed after extending the size of the hernia ring to allow reduction of the colon into the abdominal cavity but extending the hernia ring on the perineum may pose further risks to the animals.

Closure of the hernia ring may be performed by either traditional herniorrhaphy (Rayhanabad et al., 2009; Reddan, 2014), or internal obturator muscle transposition (Vnuket al., 2008). Other approaches are superficial gluteal muscle transposition, or semitendinosus muscle transposition (Mortari et al., 2005). Traditional herniorrhaphy was considered sufficient to correct the hernia ring due to low tension formed from suture at the ventral aspect of herniorrhaphy.
There are several modalities for the surgical management of megacolon, including subtotal colectomy and colotomy. The distended colon serosa and mucosa layer were assessed as viable and salvageable, rendering colectomy and subtotal colectomy of minimal value in this case. Attachment of the seromuscular layer of the colon to the abdominal wall prevents prolapse of the large intestine should pathological opening in the thoracic or abdominal cavity happens in future. Colon functions in water reabsorption from formed faeces. Colotomy predisposes the animal to incisional dehiscence and colon wall rupture, thereby rendering the procedure risky. Colopexy was performed to provide a direct route for the stool transit within the colon and also removal of the angle between damaged colon segments and a healthy portion at the pelvic inlet (Abedi et al., 2012)

Healing of intestine takes a long time; therefore, the restriction of feeding prevents the production of faeces within the large intestine and distension of the colon, which could rupture the colonic suture site. Ceftriaxone was administered to prevent secondary bacterial infection due to the manipulation of the colon, which could result in contamination from the intestinal contents onto the surrounding abdominal organs (Dietrich et al., 2002). Nutriplus gel ${ }^{\circledR}$ Virbac, Australia) was administered as an energy and vitamin supplement to the dog to improve the nutritional intake.

\section{Conclusion}

A perineal hernia can develop secondarily to megacolon and vice versa and can be acquired from nerve damage resulting from trauma (Machado et al., 2020). The condition requires emergency surgical intervention and is treatable via perineal herniorrhaphy, colostomy, and colopexy. This was successfully accomplished in this case report.

\section{Conflict of Interest}

The authors declare that they do not have any conflict of interest.

\section{Author's Contribution}

OIA, CMA, NSR and BPDA., were all involved in the conceptualization, clinical assessment, manuscript writing and transformation as well as data curation.

\section{REFERENCES}

Abedi, G., Asghari, A., Alizadeh, R.and Shayan, N. (2012). Colon Surgical Stabilization on Psoases Muscles for Treatment of Megacolon in Dog. Glob Vet. 9(2): 232-236.

Dietrich, E.S., Bieser, U., Frank, U., Schwarzer, G. and Daschner, F.D. (2002). Ceftriaxone versus other cephalosporins for perioperative antibiotic prophylaxis: A meta-analysis of 43 randomized controlled trials. Chemotherapy; 48(1): 49-56.

Fossum, T.W. (2018). Small Animal Surgery, fifth edition: Elsevier Health Sciences, Philadelphia pp 331.

Lee, A.J. et al. (2012). Use of canine small intestinal submucosa allograft for treating perineal hernias in two dogs. J. Vet. Sci. 13(3): 327-330. 
Machado, Â. V. d. L. P., Lugoch, G., dos Santos, A. P. I., Gonçalves, M. E. P., de Oliveira, M. T., Viela, J. A. P. and Beckmann, B. V. (2020). Perineal Hernia in a Bitch. Acta Sci. Vet. 48: 1-5.

Mortari, C.A., Rahal, S.C., Resende, L.A., Dal-pai-silva, M., Mamprim, M.J., Corrêa, M.A. and Antunes, S.H. (2005). Electromyographical, Ultrasonographical and Morphological Modifications in Semitendinous Muscle after Transposition as Ventral Perineal Muscle Flap. J. Vet. Med. 52(7): 359-365.

Rayhanabad, J., Sassani, P.and Abbas, M. (2009). Laparoscopic Repair of Perineal Hernia. JSLS-J Soc Laparoend, 13(2): 237-241.
Reddan, S. (2014). Herniorrhaphy of a unilateral perineal hernia and castration. Vet.Nurs. J., 29(1): 14-16.

Segev, G., Nivy, R., Kass, P.H., and Cowgill, L.D. (2013). A Retrospective Study of Acute Kidney Injury in Cats and Development of a Novel Clinical Scoring System for Predicting Outcome for Cats Managed by Haemodialysis. J.Vet. Intern. Med. 27(4): 830839.

Prokić, B., Todorović, V., Mitrović, O., Vignjević, S.and Savić-Stevanović, V. (2010). Etiopathogenesis, diagnosis and therapy of acquired megacolon in dogs. Acta Vet. 60(2-3): 273-284.

Vnuk, D., Lipar, M., Matičić, D., Smolec, O., Pećin, M.and Brkić, A. (2008). Comparison of standard perineal herniorrhaphy and transposition of the internal obturator muscle for perineal hernia repair in the dog. Vet. Archiv. 28: 197-208. 